\title{
Time Resolved Measurements of the Switching Trajectory of Pt/Co Elements Induced by Spin-Orbit Torques
}

\author{
M. M. Decker, ${ }^{1}$ M. S. Wörnle, ${ }^{1}$ A. Meisinger, ${ }^{1}$ M. Vogel,${ }^{1}$ H. S. Körner, ${ }^{1}$ \\ G. Y. Shi, ${ }^{2}$ C. Song, ${ }^{2}$ M. Kronseder, ${ }^{1}$ and C. H. Back ${ }^{1}$ \\ ${ }^{1}$ Department of Physics, Regensburg University, 93053 Regensburg, Germany \\ ${ }^{2}$ Key Laboratory of Advanced Materials (MOE), School of Materials Science and Engineering, \\ Tsinghua University, Beijing 100084, China \\ (Received 14 March 2017; published 22 June 2017)
}

\begin{abstract}
We report the experimental observation of spin-orbit torque induced switching of perpendicularly magnetized $\mathrm{Pt} / \mathrm{Co}$ elements in a time resolved stroboscopic experiment based on high resolution Kerr microscopy. Magnetization dynamics is induced by injecting subnanosecond current pulses into the bilayer while simultaneously applying static in-plane magnetic bias fields. Highly reproducible homogeneous switching on time scales of several tens of nanoseconds is observed. Our findings can be corroborated using micromagnetic modeling only when including a fieldlike torque term as well as the Dzyaloshinskii-Moriya interaction mediated by finite temperature.
\end{abstract}

DOI: 10.1103/PhysRevLett.118.257201

Magnetization switching induced by spin-orbit torques (SOTs) generated by in-plane (IP) current pulses in ferromagnetic (FM)/heavy metal (HM) bilayers has attracted great attention in recent years [1-17]. A typical structure comprises a FM element with perpendicular magnetization structured on top of a HM conductor carrying the current. Technologically, such a device has the advantage that the write current causing magnetization switching does not have to pass through a potential memory element itself, thus avoiding its degradation [18]. Studying magnetization dynamics in such elements is of interest since the exact mechanisms enabling deterministic magnetization reversal remain to be disentangled. SOT driven magnetization reversal in HM/FM bilayers originates from a combination of effects which manifest themselves as field- and dampinglike torques. These torques arise from bulk and interface effects such as the bulk spin Hall effect (SHE) or the interfacial inverse spin Galvanic effect. Recent efforts have been dedicated to the understanding of the switching process induced by static or quasistatic currents $[1,2,4,5,10,15,19]$. However, the nature of the switching process itself is still under debate. Two possible scenarios exist: coherent rotation [4] and domain nucleation and propagation [2]. The critical current densities required for these distinct processes differ by orders of magnitude since, for domain driven reversal, a much smaller energy barrier needs to be overcome. It is believed that, for devices much larger than one domain wall width, the quasistatic switching process is domain driven $[2,5,9,15]$. However, when reducing the size, it has been demonstrated recently that the switching process can be described by uniform motion [14]. By studying switching probabilities using short current pulses of variable width $[3,6,7,14]$, reliable switching for applied pulse widths as short as 180 ps [6] has been demonstrated. In these experiments, switching dynamics has been investigated indirectly by examining the final state long after the current pulse has been applied. To understand the speed and type of the SOT induced switching process in detail, temporal and spatial resolution is required which is met in this Letter using time resolved scanning magnetooptical Kerr micoscopy (TRMOKE).

Here, we measure the trajectory of the magnetization of perpendicularly magnetized $\mathrm{Pt} / \mathrm{Co}$ elements during reversal using a pump-probe approach. We observe magnetization reversal on a time scale of tens of nanoseconds mediated by domain wall motion. By comparison with micromagnetic simulations, we identify the importance of both the Dzyaloshinskii-Moriya interaction (DMI) and the fieldlike torque for the switching process.

$2 \times 2 \mu \mathrm{m}^{2}$ Co squares on top of a $2 \mu \mathrm{m}$ wide, $20 \mu \mathrm{m}$ long $\mathrm{Pt}$ line are prepared from a stack of $\mathrm{Ta}(3 \mathrm{~nm}) / \mathrm{Pt}(8.5 \mathrm{~nm}) /$ $\mathrm{Co}(0.5 \mathrm{~nm}) / \mathrm{Al}_{2} \mathrm{O}_{3}(5 \mathrm{~nm})$ grown onto thermally oxidized, highly resistive silicon by molecular beam epitaxy. The square is integrated into a $50 \Omega$ matched Au microstrip to ensure a good transmission of short current pulses. To study the dependence of size and shape, samples with $750 \mathrm{~nm}$ diameter disks have been prepared on top of the $2 \mu \mathrm{m}$ wide Pt line. The results obtained for the two geometries do not differ significantly [20].

To calibrate the TRMOKE experiments, we record hysteresis loops in the center of a $2 \times 2 \mu \mathrm{m}^{2}$ square sample in a not time resolved measurement, but by using the same laser system. Figure 1(c) shows two measurements performed statically. The upper panel shows a typical hysteresis curve. The lower panel shows quasistatic current induced switching in an applied IP field of $B_{x}=-20 \mathrm{mT}$. The input current is a square signal of $0.5 \mathrm{~Hz}$ with a current density of $\pm 4 \times 10^{10} \mathrm{~A} / \mathrm{m}^{2}$. Both measurements give access to the 


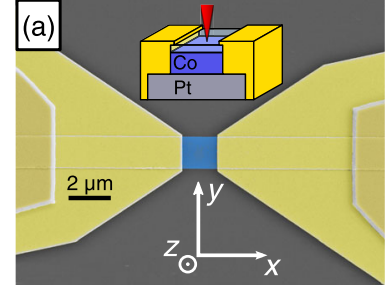

(c)
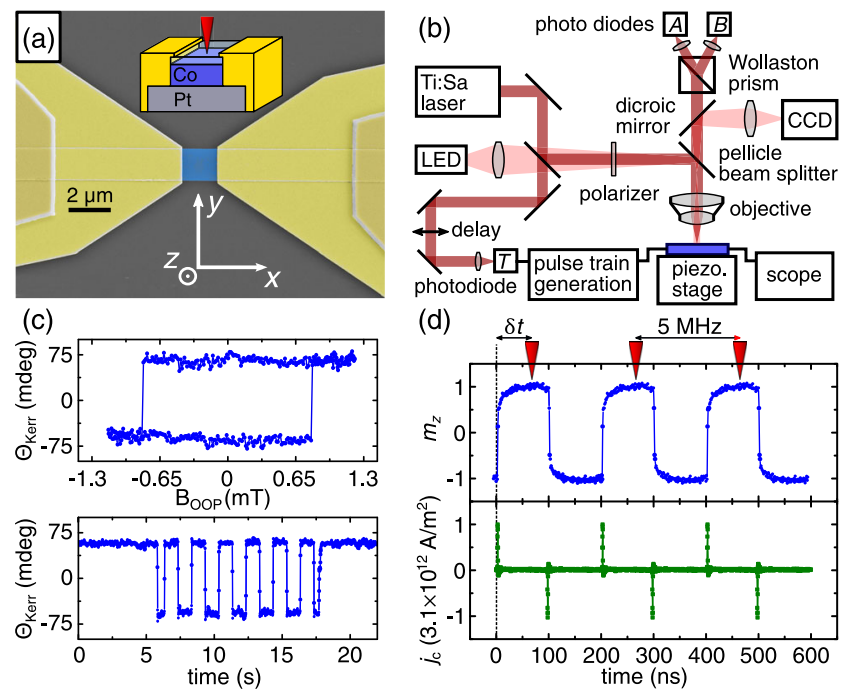

(d)
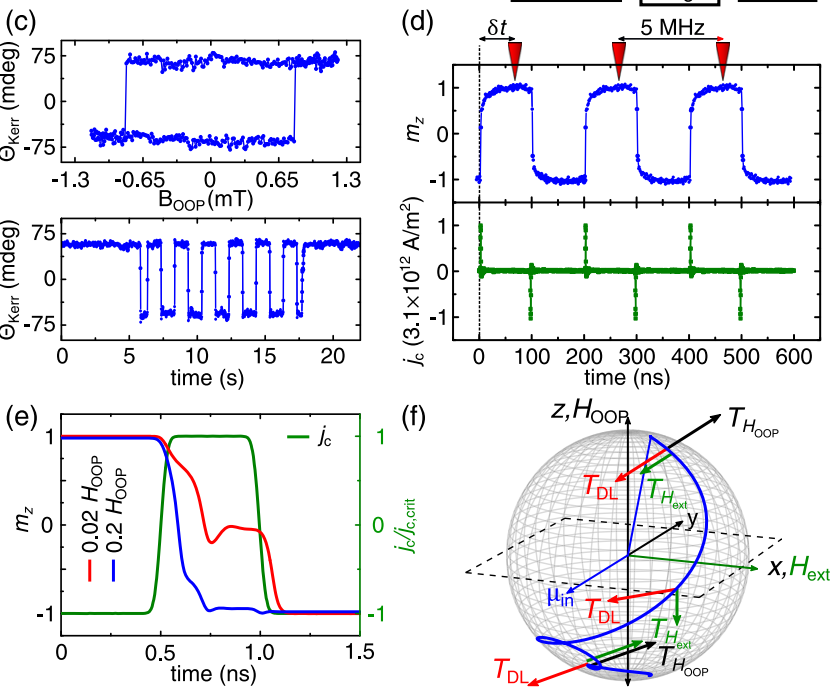

FIG. 1. (a) Colored scanning electron microscopy image of the device. The $2 \times 2 \mu \mathrm{m}^{2} \mathrm{Pt} / \mathrm{Co}$ element is marked in blue. The inset sketches the layer system. (b) A simplified block diagram of the experimental setup. (c) Static polar Kerr measurements recorded in the center of the element. (Upper panel) Hysteresis curve. (Lower panel) Quasistatic current induced magnetization reversal for $B_{x}=-20 \mathrm{mT}$ and $j_{\mathrm{Pt}}= \pm 4 \times 10^{10} \mathrm{~A} / \mathrm{m}^{2}$. (d) Time resolved measurement of magnetization reversal for $B_{x}=$ $-50 \mathrm{mT}$. (Lower panel, green curve) Current pulse train as transmitted through the sample. (Upper panel, blue curve) TRMOKE signal obtained by scanning the delay $\delta t$ between the current and laser pulses. The signal is recorded for one period (200 ns) and is repeated to elucidate the measurement technique. (e),(f) Numerical solution of the LLG equation for a $500 \mathrm{ps}$ current pulse (green curves) creating a SOT strong enough to pull the magnetization into the plane. Two cases for different external fields are shown, $H_{x}=+0.02 H_{\mathrm{OOP}}$ (red curves) and $H_{x}=$ $+0.2 H_{\mathrm{OOP}}$ (blue curves). (e) shows the $z$ component only, while (f) shows the $3 \mathrm{D}$ trajectory.

absolute Kerr signal of about 150 mdeg resulting from complete magnetization reversal enabling calibration of the signals acquired in time resolved measurements.

Magnetization dynamics is measured using a pumpprobe TRMOKE experiment with $\approx 500 \mathrm{~nm}$ resolution, as sketched in Fig. 1(b) and described in detail in Ref. [20]. In this stroboscopic experiment, the current pulse train of alternating positive and negative pulses is generated using two pulse generators which are triggered by the laser pulses and by combining their outputs. The resulting pulse train is then amplified by a broadband amplifier. Using a combination of electrical and optical delay lines, we adjust the delay between probing pulse and current pulse at the position of the sample with a timing jitter of $\sim 50 \mathrm{ps}$ over a full period of 200 ns, as shown in Fig. 1(d). Here, 1 ns wide pulses with a peak current density of $j_{\max }=3.1 \times$ $10^{12} \mathrm{~A} / \mathrm{m}^{2}$ are used to switch the magnetization in a static magnetic field of $B_{x}=-50 \mathrm{mT}$. Starting from the "down state," the positive pulse toggles the magnetization to the "up state" and the negative pulse back down, as expected for the layer sequence and a positive spin Hall angle in Pt.

The switching process of a perpendicularly magnetized ferromagnet can be understood when solving the LandauLifshitz-Gilbert (LLG) equation $[2,4,13,21]$

$$
\begin{aligned}
\frac{\partial \mathbf{m}}{\partial t}= & -\gamma \mathbf{m} \times \mu_{0} \mathbf{H}_{\mathrm{eff}}+\alpha \mathbf{m} \times \frac{\partial \mathbf{m}}{\partial t} \\
& +\gamma \tau_{\mathrm{DL}} \mathbf{m} \times(\mathbf{m} \times \mathbf{y})-\gamma \tau_{\mathrm{FL}} \mathbf{m} \times \mathbf{y} .
\end{aligned}
$$

Here, $\mathbf{m}$ denotes the unit vector of the magnetization, $\gamma=$ $180 \mathrm{rad} /(\mathrm{Ts})$ is the gyromagnetic ratio, and $\alpha$ is the Gilbert damping parameter. $\boldsymbol{H}_{\text {eff }}$ is the effective field given by the vector sum of the externally applied magnetic field $\boldsymbol{H}_{\text {ext }}$ and the effective out-of-plane (OOP) field $\boldsymbol{H}_{\mathrm{OOP}}=$ $\left[2 K^{\perp} /\left(\mu_{0} M_{s}\right)-M_{s}\right] m_{z}$, with the saturation magnetization $M_{s}$ and the uniaxial perpendicular anisotropy constant $K^{\perp}$. The effect of the SOTs is taken into account via the last two terms, which are called damping- $\left(\boldsymbol{T}_{\mathrm{DL}}\right)$ and field- $\left(\boldsymbol{T}_{\mathrm{FL}}\right)$ like SOTs, respectively. In the picture of the SHE, the strength of the dampinglike torque can be expressed as $\tau_{\mathrm{DL}}=\hbar /(2|e|) \Theta_{\mathrm{eff}} j_{c} /\left(M_{s} d_{F}\right)$, with the effective spin Hall angle $\Theta_{\text {eff }}[2,13]$, the ferromagnetic layer thickness $d_{F}$, and the injected current density $j_{c} \| \boldsymbol{x}$. For $j_{c}>0$, the injected moment $\boldsymbol{\mu}_{\text {in }}$ points in the $\mathbf{- y}$ direction for our geometry [22]. In the case of a full micromagnetic simulation, $\boldsymbol{H}_{\text {eff }}$ also includes the exchange interaction $\left(A=10^{-11} \mathrm{~J} / \mathrm{m}\right)$, and an additional DMI term which is known to be present in asymmetrically sandwiched $\mathrm{Pt} / \mathrm{Co} /$ oxide layers and is essential for reproducible domain formation [23]. For deterministic bipolar switching, the combination of a dampinglike SOT and an additional IP field perpendicular to $\mathbf{y}$ is crucial $[2,6]$. The IP field breaks the symmetry of the magnetic response to $\boldsymbol{T}_{\mathrm{DL}}$ since it exerts a torque to $\mathbf{m}$ that adds to $\boldsymbol{T}_{\mathrm{DL}}$ in one half sphere and counteracts it in the other half sphere.

In the simplest case, i.e., for coherent rotation and neglecting $\boldsymbol{T}_{\mathrm{FL}}$, the switching current can be determined analytically from $\tau_{\mathrm{DL}, \text { crit }}=\left(\mu_{0} / 2\right)\left(H_{\mathrm{OOP}}-\sqrt{2} H_{\mathrm{ext}}\right)[4,6]$. In this case, the dynamics can be calculated by numerically solving the LLG for a given set of parameters. Examples are shown in Figs. 1(e) and 1(f) for a 500 ps long pulse and two different IP fields $H_{x}=0.02 \times H_{\mathrm{OOP}}$ (the red curves) and $H_{x}=0.2 \times H_{\mathrm{OOP}}$ (the blue curves). Figure 1(e) shows that the magnetization switches within the first $100 \mathrm{ps}$ (250 ps) for the larger (smaller) field value. The dampinglike torque is chosen such that $\tau_{\mathrm{DL} \text {,max }}=\left(\mu_{0} H_{\mathrm{OOP}} / 2\right)$ and $\alpha$ is set to 0.5 [23,24]. In Fig. 1(f) the 3D trajectory of $\mathbf{m}(t)$ for the larger field value is shown, whereas in Fig. 1(e) the 
time evolution of $m_{z}$ is plotted. In Fig. 1(f) the torques resulting from the fields and the SOT are shown to elucidate the role of the IP field. Note that the switching time itself strongly depends on the strength and rise time of the pulse, as was worked out in Ref. [13].

Simplified macrospin calculations are illustrative, but they fail to explain the switching process quantitatively and even qualitatively, as we will detail below. This can have multiple origins. (i) A nonzero fieldlike torque $\boldsymbol{T}_{\mathrm{FL}}$ reduces the critical current; the strength and sign of this torque strongly influence the dynamics $[13,14]$. (ii) It is known that switching is thermally assisted. The influence of thermal fluctuations leads to a strong dependence of the critical current on the pulse length. Note that thermal activation can be included in a macrospin calculation [10,25-27]. (iii) For magnetic elements with dimensions large enough to accommodate a domain wall, the reversal process can be driven by domain nucleation and propagation, further reducing the energy barrier. For a Pt/Co bilayer, this has first been described in Ref. [2], where a model is used that implicitly includes thermal activation and domain driven switching for static currents. Subsequently, experiments using pulsed currents of variable pulse width $\tau_{p}$ have been reported discussing the effects of thermal activation $[3,6,8,10,14]$ and device size [14] on the switching mechanism.

To gain insight into the mechanisms at work, we study the reversal process as a function of time. In Fig. 2 the Kerr signal recorded in the middle of the element is displayed. The left graphs show the full recorded time span of $25 \mathrm{~ns}$; the right ones are enlarged to the first 5 ns only. Figure 2(a) shows the time evolution of $m_{z}$ for different applied fields where $H_{\text {ext }}<H_{\mathrm{OOP}}$. The fixed current pulse shown in green reaches a peak current density $j_{\max }=3.1 \times 10^{12} \mathrm{~A} / \mathrm{m}^{2}$, with $\tau_{p}=1 \mathrm{~ns}$. The switching process is slowest for the lowest field and speeds up as the field is increased, as expected from theory. For field values when $H_{\text {ext }} \gg H_{\text {crit }}$, the magnetization switches back to the initial equilibrium position $m_{z}=-1$ subsequent to the pulse. In Fig. 2(b) the pulse width is varied between $600 \mathrm{ps}$ and $1 \mathrm{~ns}$ for a fixed $B_{\text {ext }}=-50 \mathrm{mT}$ and an almost constant $j_{\max }$. For $\tau_{p}<1 \mathrm{~ns}$ the critical current density cannot be reached, leading to a fast decrease of the switching probability. This is seen as a reduced "up level," e.g., for $\tau_{p}=770 \mathrm{ps}$ where the magnetization switches with about $80 \%$ probability and the signal recorded is $0.8 m_{z}-$ $0.2 m_{z}=0.6 m_{z}$ due to statistical averaging of the pumpprobe technique. For even shorter pulses where $\tau_{p} \leq 700 \mathrm{ps}$, we observe only a small canting of $\mathbf{m}$ from the equilibrium state and subsequent relaxation. Similar results are found when reducing the peak amplitude of the current pulse at a fixed $B_{\text {ext }}=-50 \mathrm{mT}$ and $\tau_{p}=1 \mathrm{~ns}$; see Fig. 2(c). Additional measurements at high fields are shown in Ref. [20].

Making use of the spatial resolution of TRMOKE, images of the switching process are taken to investigate possible spatial inhomogeneities during reversal. An example for $\tau_{p}=1 \mathrm{~ns}, B_{\text {ext }}=-50 \mathrm{mT}$, and $j_{\max }=3.1 \times 10^{12} \mathrm{~A} / \mathrm{m}^{2}$ is shown in Fig. 3. Figure 3(a) shows the switching curve in
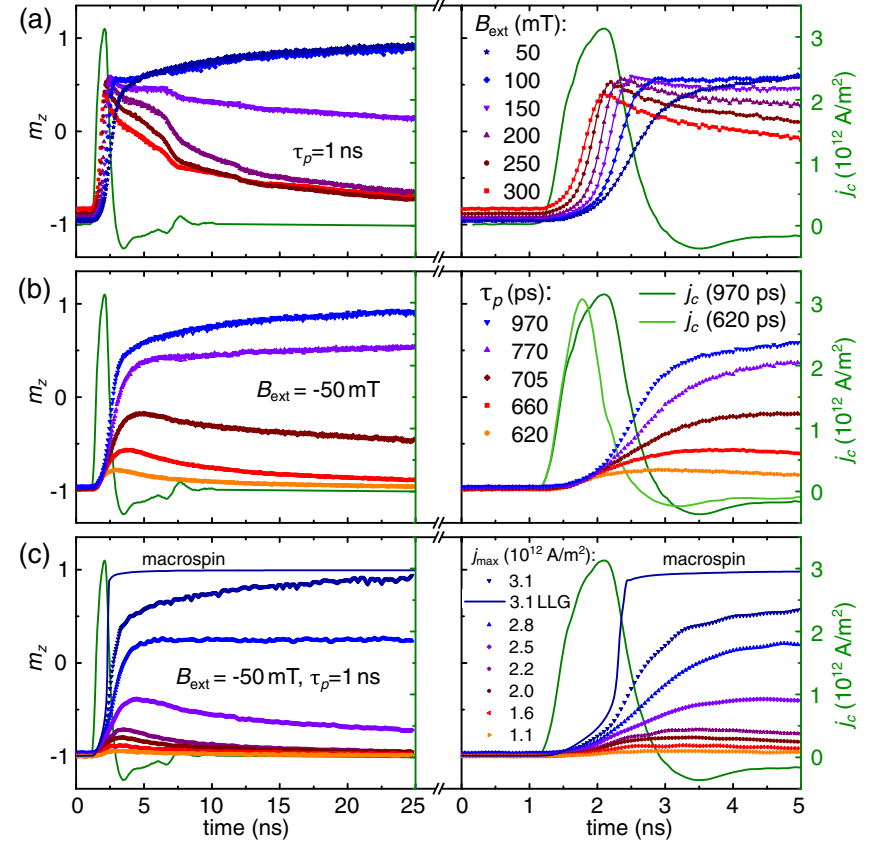

FIG. 2. Transient normalized magnetization $m_{z}(t)$ (symbols, left axis) and current density $j_{c}(t)$ (green solid lines, right axis) as a function of delay time. The right graphs show the first $5 \mathrm{~ns}$ of the respective left graphs in detail. (a) Magnetization reversal for $\tau_{p}=1 \mathrm{~ns}$ and $B_{\text {ext }}$ ranging from -50 to $-300 \mathrm{mT}$ at a fixed $j_{\max }=3.1 \times 10^{12} \mathrm{~A} / \mathrm{m}^{2}$. For large fields the magnetization relaxes back into the initial equilibrium position after the switching event. (b) Measurements for a fixed $B_{\text {ext }}=-50 \mathrm{mT}$ and different pulse durations $\tau_{p}$ ranging from 0.6 to $1 \mathrm{~ns}$. (c) Current density dependence for $\tau_{p}=1 \mathrm{~ns}$ and $B_{\text {ext }}=-50 \mathrm{mT}$. Numerical solutions of the LLG in comparison to the experiment are shown as solid lines.

blue. The inset shows the reflected intensity where the current contacts (yellow) can be seen on the left and right sides. The images shown in Fig. 3(b) are snapshots of the reversal at times marked with red dots in Fig. 3(a). The switching process is homogeneous without the appearance of propagating domains. The same result is found at different $j_{\max }$ 's and external fields. In Ref. [23], the switching of a $100 \mathrm{~nm}$ diameter disk has been simulated, and it is predicted that the switching occurs via deterministic domain nucleation at one side of the disk and subsequent domain wall propagation across the disk under the influence of the current.
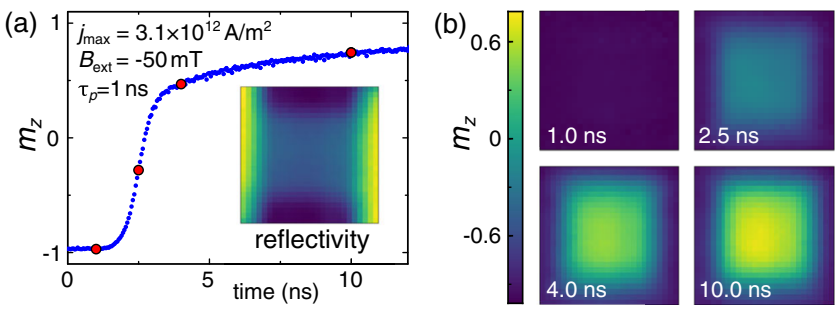

FIG. 3. (a) $m_{z}(t)$ trace recorded in the middle of the sample. The inset shows the reflectivity (topography). (b) Images of the switching process taken at distinct times shown in red in (a). 
It is also shown that the nucleation point is determined by the shape of the magnetic element in combination with the presence of DMI. Such a scenario must be visible using our experimental technique if the process is deterministic. To ensure that our results are not caused by the shape of the sample, a disk shaped sample with a diameter of $750 \mathrm{~nm}$ was measured. The results do not differ significantly from the presented data [20].

To obtain a quantitative understanding of the experimental results, Eq. (1) is solved numerically using the predetermined $M_{s}$ and $K^{\perp}$, neglecting $\boldsymbol{T}_{\mathrm{FL}}$ and thermal effects, since $\tau_{p} \leq$ $1 \mathrm{~ns}$ is below the thermally activated regime $[6,10,25,26]$. For $\tau_{p}=1 \mathrm{~ns}$ and $j_{c}=3.1 \times 10^{12} \mathrm{~A} / \mathrm{m}^{2}$, at $B_{\mathrm{ext}}=-50 \mathrm{mT}$, switching is achieved for a $\tau_{\mathrm{DL}}$ value leading to $\Theta_{\text {eff }}=0.13$, which is in good agreement with published experimental results in similar structures [6,28]; i.e. the switching current itself can, in principle, be explained by a simple LLG calculation. However, the time scale of the switching process differs from the measured data, as shown in Fig. 2(c). The solid blue lines show the numerical solution corresponding to the measured data for $j_{\max }=3.1 \times 10^{12} \mathrm{~A} / \mathrm{m}^{2}$, which is shown in dark blue symbols. In the measurement the full level $m_{z}=1$ is reached after $t \approx 30 \mathrm{~ns}$, in contrast to the much faster switching in the calculation. We emphasize that, within the macrospin model, the switching process must occur within the pulse duration due to the combination of a large value for $\alpha$ and the relatively long rise and fall times of the pulse. This statement still holds if a nonzero $\boldsymbol{T}_{\mathrm{FL}}$ or thermal activation is added to the LLG. However, $1 \mathrm{~ns}$ pulses in the $10^{12} \mathrm{~A} / \mathrm{m}^{2}$ regime are likely to heat up the element by some 10-100 K, which may significantly influence the magnetic properties, such as $M_{s}$. To estimate the impact of a temperature increase, measurements of $M_{s}(T)$ are performed [20]. A Curie temperature of $T_{C} \approx 400 \mathrm{~K}$ is found which agrees with previous reports [29]. To examine whether Joule heating of the sample could explain the long signal recovery times, finite elements COMSOL $®$ simulations are performed to investigate how rapidly the element cools down after the pulse has passed. We find that the temperature recovers its initial value in $<7 \mathrm{~ns}$ after the pulse ends. Thus, the magnetic signal should be recovered after this time. It should be noted that the peak temperature of the sample remains well below $T_{C}$ since demagnetization of the Co layer, which would manifest itself as a plateau in the Kerr data, is not observed. We conclude that a macrospin approach, even when considering Joule heating, cannot adequately explain our results.

To further investigate our finding that the reversal process appears homogeneous but does not follow a macrospin model, micromagnetic simulations are performed using $\operatorname{MUMAX}^{3}$ [30]. The material parameters used are the same as for the LLG calculations but include DMI as well. The strength of the DMI has been measured frequently in $\mathrm{Pt} / \mathrm{Co} /$ oxide systems; DMI constants range from $D d_{\mathrm{Co}} \sim$ $0.3 \mathrm{pJ} / \mathrm{m}[9,31]$ to $\sim 2 \mathrm{pJ} / \mathrm{m}$ [32-34]. In our simulations, a DMI constant of $D d_{\mathrm{Co}}=0.5 \mathrm{pJ} / \mathrm{m}$ is assumed. Larger values lead to multidomain states in zero field due to the relatively low $H_{\mathrm{OOP}}$ of our samples and the fact that DMI greatly reduces the cost of the domain walls. In Figs. 4(a) and 4(b), simulation results for two fields, -50 and $-300 \mathrm{mT}$, are shown. The two upper graphs show the averaged magnetization as a function of time. The lower panels show snapshots at distinct times marked by dots in the upper traces. In the simulation the magnetic field is tilted OOP by $1^{\circ}$ from the $x$ axis such that $H<0 \rightarrow H_{z}<0$, leading to a relaxation back into the stable equilibrium for large fields, as seen in the experiment. The similarity of the simulated and measured curves is striking. Especially interesting is the fact that the slow switching time can be reproduced for the $-50 \mathrm{mT}$ case. Most importantly we find that the experimental results can be reproduced only if DMI, a fieldlike torque $\tau_{\mathrm{FL}}$, and finite temperatures $(100 \mathrm{~K})$ are taken into account.

The result closest to the measurement is reached for $\tau_{\mathrm{FL}} / j_{c}=0.045 \mathrm{pT} \mathrm{m}^{2} / \mathrm{A}$ and $\tau_{\mathrm{DL}} / j_{c}=0.067 \mathrm{pT} \mathrm{m}^{2} / \mathrm{A}$, which is close to the literature values in [35]. The sign of $\tau_{\mathrm{FL}}$ is such that $\boldsymbol{T}_{\mathrm{FL}}$ and $\boldsymbol{T}_{\mathrm{DL}}$ try to align the magnetization in opposite directions; in this case, $\boldsymbol{T}_{\mathrm{FL}}$ destabilizes the system during the pulse. Together with the finite temperature, small domains are continuously created and annihilated during the duration of the pulse. These then relax into the new state only after the pulse is turned off. The nucleation happens in a random fashion. In the simulation, it depends on the seed of the random field used to implement temperature. If measured by a stroboscopic technique, the statistical average over many switching events results in a homogeneous value across the sample, corroborating the results shown in Fig. 3. Without DMI, this relaxation happens much faster than seen in the experiment. This scenario is observed when $\tau_{\mathrm{FL}}$ dominates over $B_{\text {ext }}$. For $\left|B_{\text {ext }}\right| \geq 100 \mathrm{mT}$, the external field stabilizes the system and speeds up the reversal process. However, owing to the low $H_{\mathrm{OOP}}$ and $H_{\text {crit }}$ in our system, the
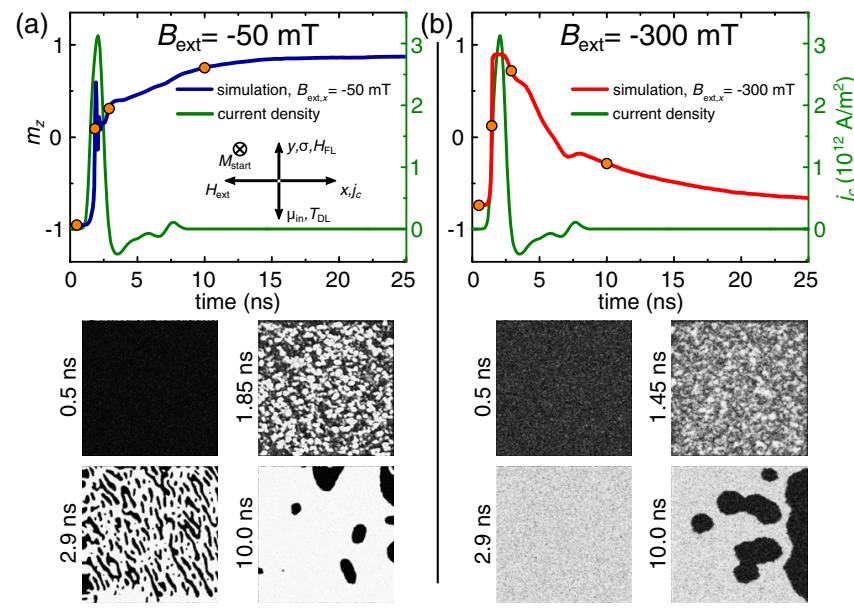

FIG. 4. Micromagnetic simulations of the switching process for $2 \mu \mathrm{m}$ wide squares using IP field values of (a) -50 and (b) $-300 \mathrm{mT}$ to model the data shown in Fig. 2(a). (Upper panels) Time evolution of $m_{\mathrm{z}}$. Orange dots denote the times for which snapshots are shown in the lower part of the figure. 
new state is not stable, such that the subsequent relaxation process overlays the switching process. For a detailed analysis of the contributions of DMI and $\tau_{\mathrm{FL}}$ (including different signs), see Ref. [20].

In conclusion, we have recorded time resolved microscopic images of the SOT induced switching process of micron sized $\mathrm{Pt} / \mathrm{Co}$ elements. We have shown that, in order to explain our results, the temperature, DMI, and fieldlike torque have to be taken into account. For the case shown here, switching occurs by the nucleation of domains. Surprisingly-and mediated by the combined action of the DMI and fieldlike torque-the switching process takes much longer than the pulse duration. Note that, for different parameter sets (particularly large OOP anisotropies), the switching process can be described by the nucleation and propagation of a domain within the current pulse duration [23].

We would like to acknowledge the DFG for funding via Grant No. SFB 689 and the group of P. Gambardella for sharing their data prior to publication.

Note added.-Recently, two papers appeared addressing similar issues $[36,37]$.

[1] I. M. Miron, K. Garello, G. Gaudin, P.-J. Zermatten, M. V. Costache, S. Auffret, S. Bandiera, B. Rodmacq, A. Schuhl, and P. Gambardella, Nature (London) 476, 189 (2011).

[2] L. Liu, O. J. Lee, T. J. Gudmundsen, D. C. Ralph, and R. A. Buhrman, Phys. Rev. Lett. 109, 096602 (2012).

[3] C. O. Avci, K. Garello, I. M. Miron, G. Gaudin, S. Auffret, O. Boulle, and P. Gambardella, Appl. Phys. Lett. 100, 212404 (2012).

[4] K.-S. K.-J. Lee, S.-W. Lee, B.-c. Min, and K.-S. K.-J. Lee, Appl. Phys. Lett. 102, 112410 (2013).

[5] S. Emori, U. Bauer, S.-M. Ahn, E. Martinez, and G. S. D. Beach, Nat. Mater. 12, 611 (2013).

[6] K. Garello, C. O. Avci, I. M. Miron, M. Baumgartner, A. Ghosh, S. Auffret, O. Boulle, G. Gaudin, and P. Gambardella, Appl. Phys. Lett. 105, 212402 (2014).

[7] M. Cubukcu, O. Boulle, M. Drouard, K. Garello, C. O. Avci, I. M. Miron, J. Langer, B. Ocker, P. Gambardella, and G. Gaudin, Appl. Phys. Lett. 104, 042406 (2014).

[8] C. Bi, L. Huang, S. Long, Q. Liu, Z. Yao, L. Li, Z. Huo, L. Pan, and M. Liu, Appl. Phys. Lett. 105, 022407 (2014).

[9] O. J. Lee, L. Q. Liu, C. F. Pai, Y. Li, H. W. Tseng, P. G. Gowtham, J. P. Park, D. C. Ralph, and R. A. Buhrman, Phys. Rev. B 89, 024418 (2014).

[10] K. S. Lee, S. W. Lee, B. C. Min, and K. J. Lee, Appl. Phys. Lett. 104, 072413 (2014).

[11] J. Torrejon, F. Garcia-Sanchez, T. Taniguchi, J. Sinha, S. Mitani, J. V. Kim, and M. Hayashi, Phys. Rev. B 91, 214434 (2015).

[12] G. Yu, P. Upadhyaya, Y. Fan, J. G. Alzate, W. Jiang, K. L. Wong, S. Takei, S. A. Bender, L.-T. Chang, Y. Jiang, M. Lang, J. Tang, Y. Wang, Y. Tserkovnyak, P. K. Amiri, and K. L. Wang, Nat. Nanotechnol. 9, 548 (2014).

[13] W. Legrand, R. Ramaswamy, R. Mishra, and H. Yang, Phys. Rev. Applied 3, 064012 (2015).
[14] C. Zhang, S. Fukami, H. Sato, F. Matsukura, and H. Ohno, Appl. Phys. Lett. 107, 012401 (2015).

[15] C. J. Durrant, R. J. Hicken, Q. Hao, and G. Xiao, Phys. Rev. B 93, 014414 (2016).

[16] S. Fukami, C. Zhang, S. DuttaGupta, A. Kurenkov, and H. Ohno, Nat. Mater. 15, 535 (2016).

[17] P. Li, T. Liu, H. Chang, A. Kalitsov, W. Zhang, G. Csaba, W. Li, D. Richardson, A. DeMann, G. Rimal, H. Dey, J. S. Jiang, W. Porod, S. B. Field, J. Tang, M. C. Marconi, A. Hoffmann, O. Mryasov, and M. Wu, Nat. Commun. 7, 12688 (2016).

[18] K. Ando, S. Fujita, J. Ito, S. Yuasa, Y. Suzuki, Y. Nakatani, T. Miyazaki, and H. Yoda, J. Appl. Phys. 115, 172607 (2014).

[19] G. Yu, P. Upadhyaya, K. L. Wong, W. Jiang, J. G. Alzate, J. Tang, P. K. Amiri, and K. L. Wang, Phys. Rev. B 89, 104421 (2014).

[20] See Supplemental Material at http://link.aps.org/ supplemental/10.1103/PhysRevLett.118.257201 for a detailed description of the measurement setup and sample design as well as for additional data and simulation results.

[21] J. Slonczewski, J. Magn. Magn. Mater. 159, L1 (1996).

[22] J. E. Hirsch, Phys. Rev. Lett. 83, 1834 (1999).

[23] N. Mikuszeit, O. Boulle, I. M. Miron, K. Garello, P. Gambardella, G. Gaudin, and L. D. Buda-Prejbeanu, Phys. Rev. B 92, 144424 (2015).

[24] S. Mizukami, E. P. Sajitha, D. Watanabe, F. Wu, T. Miyazaki, H. Naganuma, M. Oogane, and Y. Ando, Appl. Phys. Lett. 96, 152502 (2010).

[25] D. Bedau, H. Liu, J. Z. Sun, J. A. Katine, E. E. Fullerton, S. Mangin, and A. D. Kent, Appl. Phys. Lett. 97, 262502 (2010).

[26] H. Liu, D. Bedau, J. Z. Sun, S. Mangin, E. E. Fullerton, J. A. Katine, and A. D. Kent, J. Magn. Magn. Mater. 358-359, 233 (2014).

[27] M. Lederman, S. Schultz, and M. Ozaki, Phys. Rev. Lett. 73, 1986 (1994).

[28] W. Zhang, W. Han, X. Jiang, S.-H. Yang, and S. S. P. Parkin, Nat. Phys. 11, 496 (2015).

[29] T. Koyama, A. Obinata, Y. Hibino, A. Hirohata, B. Kuerbanjiang, V. K. Lazarov, and D. Chiba, Appl. Phys. Lett. 106, 132409 (2015).

[30] A. Vansteenkiste, J. Leliaert, M. Dvornik, M. Helsen, F. Garcia-Sanchez, and B. Van Waeyenberge, AIP Adv. 4, 107133 (2014).

[31] M. J. Benitez, A. Hrabec, A. P. Mihai, T. A. Moore, G. Burnell, D. McGrouther, C. H. Marrows, and S. McVitie, Nat. Commun. 6, 8957 (2015).

[32] N. H. Kim, D. S. Han, J. Jung, J. Cho, J. S. Kim, H. J. M. Swagten, and C. Y. You, Appl. Phys. Lett. 107, 142408 (2015).

[33] C. F. Pai, M. Mann, A. J. Tan, and G. S. D. Beach, Phys. Rev. B 93, 144409 (2016).

[34] M. Belmeguenai, J. P. Adam, Y. Roussigné, S. Eimer, T. Devolder, J. V. Kim, S. M. Cherif, A. Stashkevich, and A. Thiaville, Phys. Rev. B 91, 180405(R) (2015).

[35] K. Garello, I. M. Miron, C. O. Avci, F. Freimuth, Y. Mokrousov, S. Blügel, S. Auffret, O. Boulle, G. Gaudin, and P. Gambardella, Nat. Nanotechnol. 8, 587 (2013).

[36] M. Baumgartner et al., arXiv:1704.06402.

[37] J. Yoon, S. W. Lee, J. H. Kwon, J. M. Lee, J. Son, X. Qiu, K. Ji. Lee, and H. Yang, Sci. Adv. 3, e1603099 (2017). 